\title{
Thrombose und Schwangerschaft
}

\author{
Management of Thrombosis in Pregnancy
}

Autoren

Institut
S. M. Schnabl, A. Strölin

Universitäts-Hautklinik der Eberhard-Karls-Universität Tübingen

\section{Bibliografie}

DOI http://dx.doi.org/

10.1055/s-0034-1377979

Akt Dermatol 2014; 40: 514-518

(c) Georg Thieme Verlag KG

Stuttgart · New York

ISSN 0340-2541

\section{Korrespondenzadresse}

Dr. med. Saskia Maria Schnabl

Universitäts-Hautklinik,

Eberhard-Karls-Universität

Liebermeisterstr. 25

72076 Tübingen

saskia.schnabl@med.uni-

tuebingen.de

\section{Zusammenfassung \\ $\nabla$}

Eine TBVT in der Schwangerschaft geht mit erheblichen Risiken für Mutter und Kind einher. Jeder Verdacht bedarf deshalb einer sofortigen und eindeutigen Abklärung unter Berücksichtigung der Risiken für das ungeborene Kind durch die Diagnostik. Die Klinik ist häufig unspezifisch und erprobte Diagnosealgorithmen fehlen für schwangere Patientinnen.

Die Diagnostik erfolgt apparativ mittels Kompressionssonografie der proximalen und distalen Venen.

Für die Abklärung einer iliakalen Thrombose steht neben der Duplexsonografie die MR-Phlebografie zur Verfügung. Diese sollte jedoch nur bei zwin-

\section{Einleitung}

$\nabla$

Die Schwangerschaft gilt als Risikofaktor für die Entstehung venöser Thromboembolien (VTE) wie venöse Thrombosen (TBVT) und akut lebensbedrohliche Lungenarterienembolien (LE). Schwangere Frauen weisen im Vergleich zu Nichtschwangeren ein vier- bis fünffach gesteigertes Risiko für thrombotische Ereignisse auf, im Wochenbett sogar um das 20-fache. Die Inzidenz wird auf 1-2 pro 1000 Schwangerschaften geschätzt $[1,2]$. Das erhöhte Risiko besteht bereits bei Eintritt der Schwangerschaft, persistiert über den gesamten Zeitraum und steigt in den ersten Wochen postpartal nochmals an. In $80 \%$ handelt es sich um isolierte TBVT, 20\% assoziiert mit einer LE [2]. Die LE ist eine der häufigsten Ursachen der mütterlichen Morbidität und Mortalität in den industrialisierten Länder [1]. In $1,1-1,5$ pro 100000 Schwangerschaften führt eine Verlegung der Pulmonalarterie, meist verursacht durch eine TBVT, zum Tod [3].

Übliche Diagnose- und Therapiealgorithmen bei TBVT oder LE sind auf Schwangere nicht einfach übertragbar. Da die Klinik in der Schwangerschaft gender Notwendigkeit zum Einsatz kommen. Die Behandlung der Venenthrombose erfolgt in der Regel mit einem niedermolekularen Heparin $(\mathrm{NMH})$ in therapeutischer Dosisierung. Danaparoid oder Fondaparinux sind bei Heparin-induzierter Thrombozythämie als Ausweichpräparat indiziert, Fondaparinux weist hierbei eine deutlich einfachere Handhabung auf. In der Stillperiode können die Medikamente Warfarin bzw. $\mathrm{NMH}$ angewandt werden.

Die gerinnungshemmende Therapie wird bei einer Thrombose bis mindestens 6 Wochen postpartal und insgesamt über einen Zeitraum von mindestens 3 Monaten, je nach Lokalisation, fortgeführt.

oft unspezifisch ist, erfolgt die Diagnosesicherung nicht immer zeitnah. Eine unverzügliche Sicherung bzw. ein Ausschluss der Diagnose bei klinischem Verdacht ist jedoch aufgrund der schwerwiegenden Komplikationen zwingend erforderlich. Die vorliegende Arbeit beschreibt die diagnostischen und therapeutischen Schritte der TBVT in der Schwangerschaft.

\section{Pathophysiologie \\ $\nabla$}

Während der Schwangerschaft begünstigen diverse physiologische Mechanismen das Auftreten einer TBVT oder LE. Eine wesentliche Rolle spielt dabei die sogenannte Virchow-Trias mit Alteration des Endothels, Veränderung der Blutviskosität und Änderung der Strömungsgeschwindigkeit.

\section{Änderung der Strömungsgeschwindigkeit}

Hierbei stellt schon die eingeschränkte Mobilisation ein erhöhtes Thromboserisiko dar. Hinzu kommen anatomische Gegebenheiten wie zum einen die Überkreuzung der rechten Arteria iliaca 
communis durch die linke Vena iliaca communis mit mechanischer Kompression der Vene. Das Uteruswachstum führt während der Schwangerschaft zu einer Kompression der parauterinen Venen im Becken und begünstigt damit ilio-femoral lokalisierte und deszendierende Thrombosen.

Durch die Verlangsamung der Strömungsgeschwindigkeit können proximale TBVT appositionell aszendierend wachsen und bergen ein erhöhtes Risiko symptomatischer LEs.

\section{Alteration des Endothels}

Während der Geburt wird das Risiko durch von Scherkraft verursachte Endothelschäden und eine gerinnungsfördernde große Wundfläche zusätzlich erhöht. Postpartal besteht zudem das Risiko einer Ovarialvenenthrombose, die sich über die Vena iliaca interna in die benachbarten Beckenvenen ausbreiten kann [4].

\section{Veränderung der Blutviskosität}

Im Blut kommt es während der Schwangerschaft zu einer Vermehrung von pro-koagulatorischen Faktoren (Faktor II, VII, VIII, $\mathrm{X}$, Fibrinogen, von Willebrand-Faktor) und einer Abnahme von Gerinnungsinhibitoren (Protein C und S) sowie zu einer Aktivitätsabnahme von Antithrombin. Als Ausdruck der erhöhten Thrombogenität sind die Aktivierungsmarker der Hämostase Prothrombinfragment, Thrombin-Antithrombin-Komplex und D-Dimere in der Schwangerschaft erhöht und steigen im Verlauf an. Diese schwangerschaftsassoziierten Veränderungen hängen mit der bevorstehenden Geburt zusammen und stellen eine evolutionsbedingte Adaptation zur Reduktion der Blutungsneigung dar $[5,6]$.

\section{Vorbestehende und transiente Risikofaktoren}

Eine weitere Erhöhung des Risikos entsteht durch vorbestehende und transiente Risikofaktoren ( Tab.1). Eine wesentliche Rolle spielen hierbei thromboembolische Ereignisse in der Vorgeschichte sowohl in der Eigen- als auch in der Familienanamnese und eine Thrombophilie. Bei 50\% der Frauen mit einer VTE während der Schwangerschaft oder im Puerperium konnte eine hereditäre oder erworbene Thrombophilie diagnostiziert werden [2].

\section{Diagnostik}

\section{Klinik}

Die eher unspezifischen Symptome einer TBVT sind in der Schwangerschaft noch schwieriger $\mathrm{zu}$ beurteilen. Atembeschwerden und/oder Schwellungen der unteren Extremitäten kommen auch bei einem normalen Schwangerschaftsverlauf häufig vor. Neu und plötzlich aufgetretene Schwellungen, Schmerzen, Überwärmung und livide Verfärbung der Beine sowie gestaute periphere Venen sollten jedoch in der Schwangerschaft immer hinsichtlich eines Vorliegens einer TBVT hinterfragt werden. Aber auch untypische Schmerzen im Rücken meist paravertebral oder im Bereich der Leistengegend können hinweisend auf eine TBVT sein, werden in der SS jedoch häufig fehlgedeutet [7]. Da es keine zuverlässigen Diagnosealgorithmen in der Schwangerschaft gibt, gilt es unter Beachtung der Anamnese, körperlicher Untersuchungsbefunde und des Vorliegens von Risikofaktoren das weitere diagnostische Procedere zur Abklärung einer TBVT festzulegen.

Um die klinische Wahrscheinlichkeit unter diesen erschwerten Bedingungen einschätzen zu können, wird der Wells Score angewandt ( Tab.2) $[8,9]$. Da hier nur kleine Kohortenstudien, ins-
Tab. 1 Risikofaktoren für TBVT und LE während der Schwangerschaft und postpartal [4].

\begin{tabular}{|c|c|}
\hline Bestehende Faktoren & Transiente Faktoren \\
\hline Maternale Adipositas & $\begin{array}{l}\text { übermäßige Gewichtszunahme } \\
(>21 \mathrm{~kg})\end{array}$ \\
\hline Multiparität & Hyperemesis gravidarum \\
\hline $\begin{array}{l}\text { Hereditäre und erworbene } \\
\text { Thrombophilien }\end{array}$ & Dehydratation \\
\hline $\begin{array}{l}\text { Thrombose oder Thrombophlebitis } \\
\text { in der Vorgeschichte }\end{array}$ & Traumata, Blutungen \\
\hline $\begin{array}{l}\text { Kardiovaskuläre Risikofaktoren (u. a. } \\
\text { Diabetes mellitus, Nikotinabusus) }\end{array}$ & Gestationsdiabetes \\
\hline $\begin{array}{l}\text { Kardiovaskuläre Erkrankungen } \\
\text { (u. a. Herzklappenerkrankungen) }\end{array}$ & $\begin{array}{l}\text { Schwangerschaftskomplika- } \\
\text { tionen (u. a. Preeklampsie, } \\
\text { protrahierter Geburtsverlauf) }\end{array}$ \\
\hline $\begin{array}{l}\text { Chronische Erkrankungen } \\
\text { (u. a. Lupus erythematodes, } \\
\text { Antiphospholipid-Syndrom, } \\
\text { chronisch venöse Insuffizienz) }\end{array}$ & $\begin{array}{l}\text { Operationen während der } \\
\text { Schwangerschaft (v.a. Sectio) }\end{array}$ \\
\hline $\begin{array}{l}\text { Myeloproliferative Erkrankungen } \\
\text { (z.B. Polyzythaemie vera) }\end{array}$ & Schwere systemische Infektion \\
\hline \multicolumn{2}{|l|}{$\begin{array}{l}\text { Heparin-induzierte Thrombo- } \\
\text { zytopenie }\end{array}$} \\
\hline \multicolumn{2}{|l|}{$\begin{array}{l}\text { Nierenerkrankungen } \\
\text { (nephrotisches Syndrom) }\end{array}$} \\
\hline Alter $>35$ Jahre & \\
\hline
\end{tabular}

Tab.2 Wells Score zur Beurteilung der klinischen Wahrscheinlichkeit einer TBVT [8].

\begin{tabular}{|ll}
\hline Wells Score für die tiefe Beinvenenthombose & \\
\hline Klinische Merkmale & Punkte \\
\hline $\begin{array}{l}\text { Aktive maligne Tumorerkrankung } \\
\text { (oder in den letzten } 6 \text { Monaten behandelt) }\end{array}$ & +1.0 \\
\hline $\begin{array}{l}\text { Lähmung oder Immobilisation der unteren Extremitäten } \\
\text { Bettruhe (>3 Tage) oder größere Operation }\end{array}$ & +1.0 \\
$\begin{array}{l}\text { in den letzten 12 Wochen } \\
\text { Eindrückbares Ödem am symptomatischen Bein }\end{array}$ & +1.0 \\
\hline $\begin{array}{l}\text { Schwellung des gesamten Beins } \\
\text { Entlang der Beinvenen lokalisierte Schmerzen }\end{array}$ & +1.0 \\
\hline $\begin{array}{l}\text { Umfangsdifferenz des Unterschenkels >3cm } \\
\text { im Seitenvergleich }\end{array}$ & +1.0 \\
\hline $\begin{array}{l}\text { Erweiterte oberflächliche Kollateralvenen } \\
\text { auf der betroffenen Seite (keine Varizen) }\end{array}$ & +1.0 \\
\hline $\begin{array}{l}\text { TVT in der Vorgeschichte } \\
\text { Alternative Diagnose mindestens ebenso wahrscheinlich } \\
\text { wie TVT }\end{array}$ & +2.0 \\
\hline \begin{tabular}{l} 
Klinische Wahrscheinlichkeit \\
\hline
\end{tabular} & $<2:$ gering \\
\hline
\end{tabular}

besondere für das Vorliegen einer LE [10] existieren, ist die Aussagekraft dieses Scores für die TBVT in der Schwangerschaft jedoch fraglich.

Labor. Der sonst als Baustein in der Thrombosediagnostik genutzte Laborwert D-Dimer, der bei der Auflösung von quervernetztem Fibrin durch Plasmin entsteht, ist mit zunehmender Schwangerschaftsdauer oft physiologisch erhöht und oberhalb des festgelegten Referenzwerts von $<0,5 \mathrm{mg} / \mathrm{l}$ [11,12]. Zudem kann bei einer hohen klinischen Wahrscheinlichkeit ein unauffälliger Laborwert eine Verlegung insbesondere der peripheren Strombahn nicht mit letzter Sicherheit ausschließen [13]. Hämatome oder Entzündungen, wie sie vor allem postpartal auftreten, führen ebenfalls zu einem Anstieg des Laborwerts. Mit Kenntnis 


\begin{tabular}{|c|c|}
\hline $\begin{array}{l}\text { Niedriges Risiko }(0-3 \%) \\
\text { Z. n. risikoass. venöser Thrombose, nicht hormonassoziiert } \\
\text { Thrombophilie ohne Thrombose } \\
\text { (mit Ausnahmen) }{ }^{1}\end{array}$ & $\begin{array}{l}\text { Aufklären } \\
\text { Umgehende Diagnostik bei Beschwerden } \\
\text { Kompressionsstrümpfe } \\
\text { NMH bei zusätzl. Risikofaktoren }\end{array}$ \\
\hline $\begin{array}{l}\text { Mittleres Risiko (4-20\%) } \\
\text { Z.n. spontaner venöser Thrombose } \\
\text { Z.n. venöser Thrombose + Thrombophilie } \\
\text { Z.n. hormonassoziierter Thrombose }\end{array}$ & $\begin{array}{l}\text { Gabe von NMH } \\
50 \text { - } 100 \text { antiXa/kg/Tag ab dem } 1 \text {. Trimenon }\end{array}$ \\
\hline $\begin{array}{l}\text { Hohes Risiko (ca. } \mathbf{2 0 - 5 0 \% )} \\
\text { Antiphospholipidsyndrom } \\
\text { Antithrombin-Mangel mit Thrombosen } \\
\text { Akute venöse Thrombose/Embolie } \\
\text { Mulitple venöse Thrombosen in der Vorgeschichte }\end{array}$ & $\begin{array}{l}\text { Gabe von NMH } \\
100-200 \text { antiXa/kg/Tag ab dem 1. Trimenon }\end{array}$ \\
\hline
\end{tabular}

Tab. 3 Risiko bei vorangegangener Thrombose oder Thrombophilie und entsprechende Indikation zur Thromboseprophylaxe bei Risikopatientinnen [20]. dieser physiologischen Laborschwankungen kann der D-DimerWert bei Verdacht auf eine Verlegung in der Beckenetage aber trotzdem von Nutzen sein. Die Beckenetage ist bei fortgeschrittener Schwangerschaft häufig duplexsonografisch nicht gut einsehbar. Besteht nach Ausschluss einer TBVT des Ober- und Unterschenkels der Verdacht auf eine Verlegung der Beckenetage, können die D-Dimere unter Beachtung eines trimesterabhängigen höheren D-Dimer-Frequenzwertes hilfreich sein und die Sensitivität und Spezifität erhöhen [14].

Eine Thrombophilie konnte bei 50\% der Frauen nachgewiesen werden, die in der Schwangerschaft eine TBVT oder LE entwickelten. Dies hat zwar zunächst in der Schwangerschaft keine therapeutische Konsequenz, kann aber auf längere Sicht, insbesondere für prophylaktische Maßnahmen, z.B. bei einer weiteren Schwangerschaft eine Relevanz haben ( $\bullet$ Tab.3). Ein Thrombophiliescreening ist deshalb im Verlauf ratsam [5,2]. Aufgrund der Komplexität der Bedeutung der Thrombophilie in der Schwangerschaft wird in dieser Arbeit auf die Einzelheiten der Thrombophilie nicht näher eingegangen [15].

\section{Bildgebende Diagnostik}

Zur Diagnostik eines thrombembolischen Geschehens in der Schwangerschaft besteht kein validierter Diagnostikalgorithmus. Als Untersuchungsmethode der Wahl zur Diagnose einer TBVT gilt auch in der Schwangerschaft die farbkodierte Kompressionsduplexsonografie. Mit dieser Untersuchungsmethode lassen sich thrombotische Ablagerungen und deren Beschaffenheit in den Venen beurteilen. Des Weiteren lassen sich wichtige Differenzialdiagnosen der TBVT (z. B. Bakerzyste, Hämatome usw.) durch die sonografische Darstellung benachbarter Strukturen erkennen. Grundsätzlich basiert die Untersuchungsmethode auf der Frage, ob das Gefäßlumen durch Druck mit dem Schallkopf zu komprimieren ist. Das Lumen einer normalen Vene lässt sich mit dem Schallkopf vollständig komprimieren, die Venenwand kann im komprimierten Zustand nicht mehr vom Umgebungsgewebe abgegrenzt werden. Liegt eine Thrombose vor, lässt sich das Lumen nur noch teilweise oder nicht mehr komprimieren. Die Untersuchung sollte vor allem im Querschnitt durchgeführt werden, bei Darstellung im Längsschnitt kann der Schallkopf seitlich abweichen [16]. Gegebenfalls kann die zusätzliche Verwendung der farbkodierten Sonografie zum B-Bild hilfreich sein, insbesondere zur Darstellung der schlecht einsehbaren Beckenvenen. Die farbkodierte Kompressionssonografie ist in der Schwangerschaft die Untersuchungsmethode der Wahl. Ihr Vorteil liegt in der Nichtinvasivität ohne Strahlen- oder Kontrastmittelexposition und der beliebigen Wiederholbarkeit zur Verlaufsbeobachtung ohne größere Risiken für Mutter und Kind. Von Nachteil ist allerdings die Untersucherabhängigkeit und erschwerte Beurteilbarkeit der Beckenvenen v.a. in der Schwangerschaft.

Zur Diagnosesicherung einer ilio-femoralen Thrombose wird die Durchführung einer Magnetresonanz-Phlebografie empfohlen. Allerdings besteht im 1. und 2. Trimenom eine Kontraindikation gegen die Verwendung von Kontrastmittel. Im 3. Trimenon kann die Untersuchung bei zwingender Notwendigkeit mit Kontrastmittel mit zyklischer Struktur durchgeführt werden $[1,4]$.

\section{Therapie \\ $\checkmark$}

Die Betreuung schwangerer Patientinnen mit einer TBVT und/ oder LE sollte interdisziplinär erfolgen. In erster Linie verfolgt die Behandlung einer TBVT in der Schwangerschaft das Ziel, die Gefahr einer LE zu minimieren und eine Thrombusaszension zu verhindern. Durch eine frühe Diagnosestellung und Therapieeinleitung können auch Spätkomplikationen wie das postthrombotische Syndrom verhindert werden. Eine unmittelbare Antikoagulation (unter Berücksichtigung der Kontraindikationen) nach Diagnosestellung, aber auch bei hoher klinischer Wahrscheinlichkeit ohne primäre Diagnosesicherung, stellt die Therapie der Wahl dar. Im zweiten Fall sollte dann die Diagnostik ohne Zeitverzögerung durchgeführt werden.

Antikoagulation. Zur Antikoagulation während der Schwangerschaft werden unfraktionierte Heparine (UFH) und niedermolekulare Heparine (NMH) ohne Bedenken eingesetzt. Es besteht keine Plazentagängigkeit und Heparine werden nur in geringer Menge und dann nicht wirksam in der Muttermilch nachgewiesen. Aufgrund der langjährigen klinischen Erfahrung können Heparine in der Schwangerschaft als sicher eingestuft werden [17]. Eine eventuell aufgrund der Gewichtsschwankungen und Zunahme des zirkulierenden Blutvolumens benötigte Steuerung der Heparine kann über die aktivierte partielle Thromboplastinzeit und Anti-Xa-Aktivität (Kontrollen 28. und 36.SSW) erfolgen. NMH sind gegenüber den UFH aufgrund der leichteren Handhabung und dem geringeren Nebenwirkungsprofil (weniger Osteoporose- und Blutungskomplikationen, niedrigeres Risiko für Heparin-induzierte Thrombozytopenie) zu bevorzugen. Eine längere Halbwertszeit und schwierige Antagonisierbarkeit ist vor allem bei geplanten operativen Eingriffen oder der bevorstehenden Geburt zu berücksichtigen [18]. Hier und bei fortgeschrittener Niereninsuffizienz stellen die UFH die Therapie der Wahl dar. Cumarine gelten in der Schwangerschaft als kontraindiziert, da sie plazentagängig sind und die Gefahr von kindlichen Blutungen und kongenitalen Fehlbildungen besteht [19]. In der Stillperiode ist eine Umstellung der Antikoagulation auf den 
Tab. 4 Pausierung der Antikoagulation bei Geburtsbeginn oder geplanter Intervention [23].

\begin{tabular}{|lll|}
\hline & $\begin{array}{l}\text { Vor Punktion/ } \\
\text { Katheter }\end{array}$ & $\begin{array}{l}\text { Nach Punktion/ } \\
\text { Katheter }\end{array}$ \\
\hline $\begin{array}{l}\text { UFH (Prophylaxe) } \\
\text { UFH (Therapie) }\end{array}$ & $4 \mathrm{~h}$ & $1 \mathrm{~h}$ \\
\hline $\mathrm{NMH}$ (Prophylaxe) & $4-6 \mathrm{~h}$ & $\begin{array}{l}1 \mathrm{~h} \text { (keine i.v. } \\
\text { Bolusgabe) }\end{array}$ \\
\hline $\mathrm{NMH}$ (Therapie) & $12 \mathrm{~h}$ & $2-4 \mathrm{~h}$ \\
\hline $\begin{array}{l}\text { Fondaparinux } \\
\text { (Prophylaxe }<2,5 \mathrm{mg} / \mathrm{d})\end{array}$ & $24 \mathrm{~h}$ & $2-4 \mathrm{~h}$ \\
\hline
\end{tabular}

Vitamin K-Antagonisten Warfarin möglich, allerdings erst ab dem 3. postnatalen Tag. Warfarin ist in der Stillperiode als unbedenklich einzustufen [20]. Für die neuen direkten Thrombin- und Faktor-Xa-Inhibitoren besteht derzeit in der Schwangerschaft keine sichere Studienlage. Danaparoid oder Fondaparinux sind nur in Ausnahmefällen wie bei Heparin-induzierter Thrombozythämie indiziert $[21,22]$.

Blutungskomplikationen treten am häufigsten während der Entbindung auf, daher sollten, wenn medizinisch vertretbar, die Heparine möglichst mit dem Wehenbeginn pausiert werden ( Tab.4; [23]). Grundsätzlich sollte bei einer frisch aufgetretenen TBVT (Ende 3.Semester) die Phase ohne Antikoagulation durch eine geplante Geburtseinleitung oder Sectio minimiert werden. Unter Nutzen-Risiko-Abwägung wird individuell eine frühestmögliche Wiedereinleitung der Antikoagulation nach der Geburt durchgeführt. Grundsätzlich wird empfohlen, nach Abklärung einer relevanten Hämorrhagie 6 Stunden nach einer natürlichen Geburt bzw. 12 Stunden nach einer Sectio wieder mit der Antikoagulation zu beginnen [24]. Aktuell fehlen genaue Daten ob, wann und um wieviel die therapeutische Dosis nach der Akutbehandlung im Verlauf der Schwangerschaft reduziert werden kann [25,26]. Empfohlen wird bei diagnostizierter proximaler TBVT oder LE die Fortführung der Antikoagulation ab der Erstdiagnose für die Dauer der Schwangerschaft sowie mindestens 6 Wochen postpartal bzw. über 6 Monate [20].

\section{Kompressionstherapie}

Die Durchführung einer konsequenten Kompressionstherapie lindert die Akutsymptome der TBVT. Im Verlauf soll sie die Entstehung eines postthrombotischen Syndroms verhindern, das sich bei $>50 \%$ der Schwangeren mit TBVT entwickelt. Betroffen sind hierbei am häufigsten Patientinnen mit proximaler und ausgeprägter Thrombose.

\section{Implantation eines Vena cava-Schirms (VCS)}

Die generelle Implantation eines VCS bei Schwangeren mit proximaler TBVT wird nicht empfohlen. Lungenembolien können damit zwar vermieden werden, haben aber keinen Einfluss auf die Kurz- oder Langzeitmortalität bei höherer Rate an Rezidivthrombosen $[27,25]$. Als Indikationen zur Implantation eines Cavafilters gelten die Kontraindikation zur Antikoagulation oder rekurrente oder progrediente VTE unter bestehender suffizienter Antikoagulation. Darüber hinaus kann die Implantation vereinzelt bei speziellen prä- und perioperativen Bedingungen gegeben sein. Das betrifft vorwiegend Schwangere mit frischer proximaler Venenthrombose (2-4 Wochen vor dem Entbindungstermin) und erhöhtem Blutungsrisiko mit passagerer Kontraindikation zur Antikoagulation oder im Umfeld von Operationen. Hierbei werden bevorzugt wiederentfernbare Systeme eingesetzt [28].

\section{Lyse}

Der Erfolg thrombusbeseitigender Maßnahmen mittels Thrombolyse, ggf. in Kombination mit Thrombektomie sowie die Katheter-gestützte pharmako-mechanische Thrombektomie bei frischen ilio-femoralen TBVT sind zwar belegt (Enden Lance 2012), deren Durchführung in der Schwangerschaft aufgrund der hohen behandlungsspezifischen Risiken (hohes Blutungsrisiko für Mutter und Kind) und der fehlenden Datenlage aber nicht zu empfehlen. Nur bei Schwangeren mit einer hämodynamisch instabilen LE sollte unter einer sorgfältigen Risiko-Nutzen-Abwägung neben einer adjuvanten Gabe von Heparin eine lebenserhaltende Lysetherapie durchgeführt werden [29].

\section{Thromboseprophylaxe in der Schwangerschaft $\nabla$}

Das Risiko einer TBVT in der Schwangerschaft ist zwar im Vergleich zur Allgemeinbevölkerung erhöht, es wird aber wegen des geringen absoluten Risikos keine generelle medikamentöse Prophylaxe empfohlen. Durch eine suffiziente Kompressionstherapie und damit verbundener Reduktion des Gesamtquerschnitts des Venensystems können jedoch klinische Beschwerden durch die Überbelastung des Venensystems reduziert werden. Dies gilt insbesondere für Patientinnen mit vorbestehender Varikose und/ oder Z.n. Phlebitiden oder Thrombosen in der Vorgeschichte. Eine Erhöhung der Flussgeschwindigkeit durch eine Kompressionstherapie in der Schwangerschaft bis in die proximalen Venen ist durch Studien hinreichend belegt [30].

Bei Patientinnen mit Thrombose oder Thrombophlebitis in der Vorgeschichte, nachgewiesener Thrombophilie und/oder vorangegangener Hormonbehandlung im Rahmen einer Kinderwunschbehandlung besteht ein erhöhtes Risiko für das Auftreten einer VTE bzw. eines Thromboserezidives. In diesen Fällen ist die

\author{
Niedriges Risiko \\ Z. n. risikoass. venöser Thrombose, nicht hormonassoziiert \\ Thrombophilie ohne Thrombose (mit Ausnahmen) \\ Mittleres Risiko \\ Z. n. spontaner venöser Thrombose \\ Z.n. venöser Thrombose + Thrombophilie \\ Z. n. hormonassoziierter Thrombose \\ Hohes Risiko \\ Antiphospholipidsyndrom hochdosiert \\ Antithrombin-Mangel mit Thrombosen \\ Akute venöse Thrombose/Embolie \\ Multiple venöse Thrombosen zuvor
}

post-partal NMH für (4 - )6 Wochen

beginnend am Abend der Entbindung

NMH absetzen bei Wehenbeginn

Zeitabstand zur Periduralanästhesie beachten

postpartal NMH für 6 Wochen

$\mathrm{NMH}$ absetzen bei Wehenbeginn oder Umstellung

auf UFH

s.c. oder i. v. PTT adjustiert

postpartal Umstellung auf Coumadin
Tab. 5 Vorgehen bei Patientinnen mit prophylaktischer Antikoagulation während der Geburt je nach Risikogruppe [20]. 
Indikation zu einer medikamentösen Thromboembolie-Prophylaxe mit niedermolekularem Heparin individuell zu ermitteln. Bewährt hat sich hier eine Einteilung in 3 Risikogruppen ( Tab.3, Tab.5; [20]). Letztlich handelt es sich um Einzelfallentscheidungen mit Einschätzung der individuellen Thrombosegefährdung und darauf aufbauender fundierter Stratefizierung zur risikoadaptierten Prophylaxe.

\section{Interessenkonflikt}

$\nabla$

Die Autoren geben an, dass kein Interessenkonflikt besteht.

\section{Abstract}

\section{Management of Thrombosis in Pregnancy}

Deep vein thrombosis in pregnancy is a considerable risk for mother and child. In case of suspected thrombosis instant distinct diagnosis is needed considering the risk for the unborn child through medical diagnostics. Symptoms are often nonspecific and there is no approved algorithm for the diagnosis of vein thrombosis in pregnancy.

Diagnostic analysis is done by duplex sonography with compression of the proximal and distal veins.

When an iliac thrombosis is suspected MR-Phlebography can be useful in addition to duplex sonography, but should only be used in urgent cases.

For the treatment of vein thrombosis low molecular heparin in therapeutic dosage is used usually. Danaparoid and Fondaparinux are indicated in patients with heparin-induced thrombocytopenia. In the nursing period low molecular heparin or warfarin can be applied.

Antikoagulant therapy for deep vein thrombosis should be continued for at least 6 weeks after birth, but at least 3 month in total depending on localization.

\section{Literatur}

1 Marik PE, Plante LA. Current Concepts: Venous Thromboembolic Disease and Pregnancy. New England Journal of Medicine 2008; 19 : $2025-2033$

2 Heit JA, Kobbervig CE, James AH et al. Trends in the incidence of venous thromboembolism during pregnancy or postpartum: A 30-year population-based study. Annals of Internal Medicine 2005; 10: 697-706

3 James AH, Jamison MG, Brancazio LR et al. Venous thromboembolism during pregnancy and the postpartum period: Incidence, risk factors, and mortality. American Journal of Obstetrics and Gynecology 2006; 5: $1311-1315$

4 Randrianarisoa E, Abele H, Balletshofer B. Diagnosis and treatment of venous thromboembolism during pregnancy and puerperium. Phlebologie 2013; 6: 315-321

5 Greer IA. Thrombosis in pregnancy: maternal and fetal issues. Lancet 1999; 9160: 1258 - 1265

6 Greer IA. The challenge of thrombophilia in maternal-fetal medicine. New England Journal of Medicine 2000; 6: 424-425

7 Ginsberg JS, Brilledwards P, Burrows RF et al. Venous Thrombosis During Pregnancy - Leg and Trimester of Presentation. Thrombosis and Haemostasis 1992; 5: 519-520

8 Wells PS, Ginsberg JS, Anderson DR et al. Use of a clinical model for safe management of patients with suspected pulmonary embolism. Annals of Internal Medicine 1998; 12: 997
9 Wells PS, Anderson DR, Rodger $M$ et al. Evaluation of D-dimer in the diagnosis of suspected deep-vein thrombosis. New England Journal of Medicine 2003; 13: 1227-1235

10 O'Connor C, Moriarty J, Walsh J et al. The application of a clinical risk stratification score may reduce unnecessary investigations for pulmonary embolism in pregnancy. Journal of Maternal-Fetal \& Neonatal Medicine 2011; 12: 1461 - 1464

11 Kline JA, Williams GW, Hernandez-Nino J. D-dimer concentrations in normal pregnancy: New diagnostic thresholds are needed. Clinical Chemistry 2005; 5: 825-829

12 Stein PD, Hull RD, Patel KC et al. D-dimer for the exclusion of acute venous thrombosis and pulmonary embolism - A systematic review. Annals of Internal Medicine 2006; 8: 589-602

13 Sivandarajah S, Horner D. Current Evidence Does Not Support the Use of A Negative D-Dimer to Rule Out Suspected Pulmonary Embolism in Pregnancy. Emergency Medicine Journal 2011; 3: 245-246

14 Chan WS, Lee A, Spencer FA et al. D-dimer testing in pregnant patients: towards determining the next 'level' in the diagnosis of deep vein thrombosis. Journal of Thrombosis and Haemostasis 2010; 5: $1004-$ 1011

15 Gonser L, Strolin A. Thromboembolic complications and the importance of thrombophilia in pregnancy. Phlebologie 2013; 6: 309-314

16 Elias A, Lecorff G, Bouvier JL et al. Value of Real-Time B-Mode Ultrasound Imaging in the Diagnosis of Deep-Vein Thrombosis of the Lower-Limbs. International Angiology 1987; 2: 175-182

17 Junqueira DRG, Carvalho MD, Perini E. Heparin-induced thrombocytopenia: a review of concepts regarding a dangerous adverse drug reaction. Revista da Associacao Medica Brasileira 2013; 2: 161 - 166

18 Greer IA, Nelson-Piercy C. Low-molecular-weight heparins for thromboprophylaxis and treatment of venous thromboembolism in pregnancy: a systematic review of safety and efficacy. Blood 2005; 2: 401-407

19 Schaefer C, Hannemann D, Meister $R$ et al. Vitamin K antagonists and pregnancy outcome - A multi-centre prospective study. Thrombosis and Haemostasis 2006; 6: 949-957

20 Bates SM, Greer IA, Middeldorp S et al. VTE, Thrombophilia, Antithrombotic Therapy, and Pregnancy Antithrombotic Therapy and Prevention of Thrombosis, 9th ed.: American College of Chest Physicians Evidence-Based Clinical Practice Guidelines. Chest 2012; 2: E691S - E736S

21 Bauersachs R, Berkowitz SD, Brenner B et al. Oral Rivaroxaban for Symptomatic Venous Thromboembolism. New England Journal of Medicine 2010; $26: 2499-2510$

22 Büller HR, Prins $M H$, Lensing $A W$ et al. Oral Rivaroxaban for the Treatment of Symptomatic Pulmonary Embolism. New England Journal of Medicine 2012; 14: 1287-1297

23 Gogarten W, van Aken H, Büttner J et al. AWMF Leitlinien Nr. 001/005: Rückenmarknahe Regionalanästhesien und Thromboembolieprophylaxe/antithrombotische Medikation. Anästh Intensivmedizin 2007; 48: $109-124$

24 Horlocker TT, Wedel DJ, Rowlingson JC et al. Regional Anesthesia in the Patient Receiving Antithrombotic or Thrombolytic Therapy American Society of Regional Anesthesia and Pain Medicine Evidence-Based Guidelines (Third Edition). Regional Anesthesia and Pain Medicine 2010; 1: 64-101

25 Hach-Wunderle $V$ et al. AWMF Leitlinie Nr. 065/002: Diagnostik und Therapie der Venenthrombose und der Lungenembolie (AWMF online 2010). Im Internet: http://www.awmf.org/leitlinien/detail/11/065-002. html; Stand:06/2010

26 Harenberg J, Heilmann L, Rath $W$ et al. Niedermolekulare Heparine in der Schwangerschaft. Deutsches Ärzteblatt 2002; 99: A424-A432

27 Hajduk B, Tomkowski WZ, Matek G et al. Vena Cava Filter Occlusion and Venous Thromboembolism Risk in Persistently Anticoagulated Patients. A Prospective, Observational Cohort Study. Chest 2009; 4: $877-882$

28 Lyon SM, Riojas GE, Uberoi R et al. A Short- and Long-term Retrievability of the Celect Vena Cava Filter: Results from a Multi-institutional Registry. Journal of Vascular and Interventional Radiology 2009; 11: $1441-1448$

29 Leonhardt G, Gaul C, Nietsch HH et al. Thrombolytic therapy in pregnancy. Journal of Thrombosis and Thrombolysis 2006; 3: 271-276

30 Buchtemann AS, Steins A, Volkert B et al. The effect of compression therapy on venous haemodynamics in pregnant women. British Journal of Obstetrics and Gynaecology 1999; 6: 563-569 